\title{
Neurobiologie
}

\section{Proteinkomplexe an inhibitorischen Synapsen in psychiatrischen Störungen}

\section{DILJA KRUEGER-BURG}

KLINIK FÜR PSYCHIATRIE UND PSYCHOTHERAPIE, UNIVERSITÄTSMEDIZIN GÖTTINGEN, UND MAX-PLANCK-INSTITUT FÜR EXPERIMENTELLE MEDIZIN, GÖTTINGEN

\section{Organizational protein complexes at inhibitory postsynapses in the brain} play a key role in regulating the flow of information through neuronal networks, and accordingly, abnormalities in these complexes can contribute to the pathophysiology of psychiatric disorders. Recent research indicates that the molecular composition of these complexes is highly diverse and synapse-specific, posing major challenges for basic research but also offering opportunities for potential clinical applications.

DOI: $10.1007 / \mathrm{s} 12268-019-0224-\mathrm{x}$ (c) Die Autorin 2019

\section{Open Access}

Das Gehirn besteht aus einem hoch komplexen Netzwerk aus Milliarden von Nervenzellen, die durch Billionen von synaptischen Verbindungen verknüpft sind, von denen jede auf ihre eigene Weise zur neuronalen Informationsverarbeitung und somit zur Entste- hung von Verhaltensmustern beiträgt. Die exzitatorischen oder erregenden Neuronen, die ihre Zielneurone aktivieren und somit eine gehirnweite Signalübertragungskette bilden. Eine mindestens ebenso wichtige Rolle Mehrheit dieser Nervenzellen zählen zu den spielen jedoch die inhibitorischen oder hemmenden Neurone, die die Erregbarkeit ihrer Zielneurone reduzieren und ihre Aktivierung verhindern [1]. Ähnlich wie die Bremsen im Auto leisten sie einen essenziellen Beitrag zur Kontrolle und Gestaltung des Informationsflusses durch das Gehirn. Es ist daher wenig überraschend, dass Veränderungen in der Funktion inhibitorischer Neurone und Synapsen wesentlich zur Entstehung von psychiatrischen Störungen wie Schizophrenie, Autismus und Angststörungen beitragen können [1, 2]. Dementsprechend ist ein besseres Verständnis der Biologie des inhibitorischen Systems unerlässlich für die Entwicklung neuer therapeutischer Ansätze für diese Erkrankungen.

\section{Signalübertragung an inhibitorischen} Synapsen

Inhibitorische Synapsen bestehen aus einer präsynaptischen und einer postsynaptischen
A

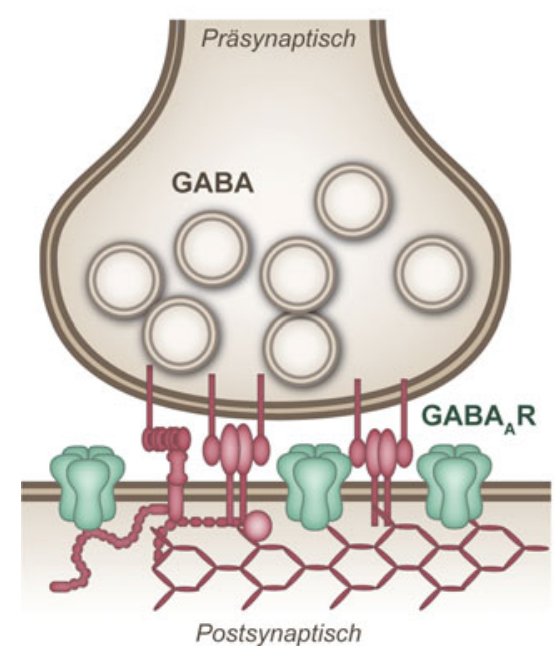

B

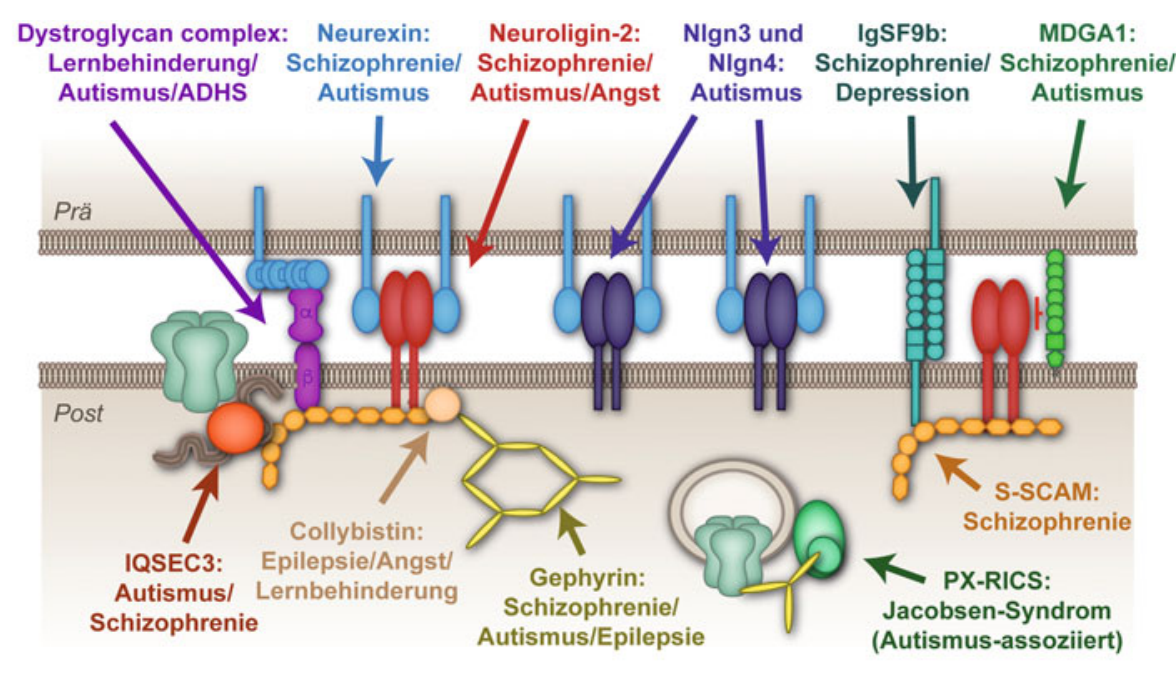

$\Delta$ Abb. 1: Proteinkomplexe an inhibitorischen Postsynapsen [3]. A, Übersicht einer inhibitorischen Synapse. Das präsynaptische Endknöpfchen setzt den Neurotransmitter GABA aus Vesikeln in den synaptischen Spalt frei. An der postsynaptischen Membran bindet GABA an GABA -Rezeptoren $^{-}$ $\left(G A B A_{A} R\right.$, grün), deren Lokalisierung und Funktion durch organisatorische Proteinkomplexe (rot) gesteuert werden. B, Assoziation von organisatorischen Proteinen an inhibitorischen Synapsen mit psychiatrischen Erkrankungen. Neurexine, Neuroligine (Nlgn), lgSF9b und der Dystroglykankomplex sind Adhäsionsproteine. MDGA1 ist ein membranständiger Inhibitor von Nlgn2. Gephyrin und S-SCAM (synaptic scaffolding molecule) sind Gerüstpro-

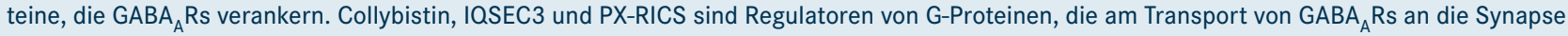
beteiligt sind. 
A Zelluläre Heterogenität

Membran, die durch den synaptischen Spalt verbunden sind. Das präsynaptische Endknöpfchen enthält kleine, mit Neurotransmitter gefüllte Vesikel, wobei GABA ( $\gamma$-Aminobuttersäure) den häufigsten inhibitorischen Neurotransmitter im zentralen Nervensystem darstellt (Abb. 1A). Wird das präsynaptische Neuron stimuliert, setzt es GABA in den synaptischen Spalt frei, wo es an postsynaptische membranständige $\mathrm{GABA}_{\mathrm{A}}$-Rezeptoren $\left(\mathrm{GABA}_{\mathrm{A}} \mathrm{R}\right)$ bindet. GABA ${ }_{\mathrm{A}}$ Rs sind Ligandenaktivierte Ionenkanäle, die für Chloridionen durchlässig sind [2]. Unter physiologischen Bedingungen wird hierdurch die postsynaptische Membran hyperpolarisiert und somit schwerer erregbar, was die hemmende Wirkung der GABAergen synaptischen Übertragung erklärt. Die Lokalisierung und Eigenschaften der $\mathrm{GABA}_{A}$ Rs an der postsynaptischen Membran werden durch ein Vielzahl regulatorischer Proteine gesteuert, darunter intrazelluläre Gerüst- und Signal-übertragende Proteine sowie transsynaptische Zelladhäsionsproteine, die eine molekulare Brücke zwischen der prä- und der postsynaptischen Seite bilden [3]. Zu den bekanntesten Komponenten dieser organisatorischen Proteinkomplexe zählen das Gerüstprotein Gephyrin, das an $\mathrm{GABA}_{\mathrm{A}}$ Rs bindet, der guanine nucleotide exchange factor Collybistin und das Adhäsionsprotein Neuroligin-2 (Nlgn2), die Gephyrin an der Synapse verankern (Abb. 1B, [4]). Lange Zeit waren Gephyrin, Collybistin und Nlgn2 nahezu die einzigen organisatorischen Proteine, von denen bekannt war, dass sie spezifisch an inhibitorischen Synapsen agieren. In den vergangenen Jahren wurde jedoch eine Vielzahl neuer Komponenten dieser Proteinkomplexe identifiziert, darunter IgSF9b (immunoglobulin superfamily member 9b), MDGA1 und 2 (MAM domain containing glycosylphosphatidylinositol anchor 1/2) sowie IOSEC3 (IQ motif and Sec7 domain 3), und inzwischen ist klar, dass die organisatorischen Strukturen erheblich umfassender und vielfältiger sind, als zunächst angenommen. Interessanterweise wurden Varianten bei vielen dieser Proteine mit diversen psychiatrischen Erkrankungen assoziiert (Abb. 1B, [3]), was die Notwendigkeit eines besseren Verständnisses der Struktur und Funktion dieser Komplexe unterstreicht.

\section{Heterogenität im inhibitorischen System}

Auffällig bei der Betrachtung dieser Assoziationen mit psychiatrischen Störungen ist, dass nicht alle Mutationen dieselben Konsequen-

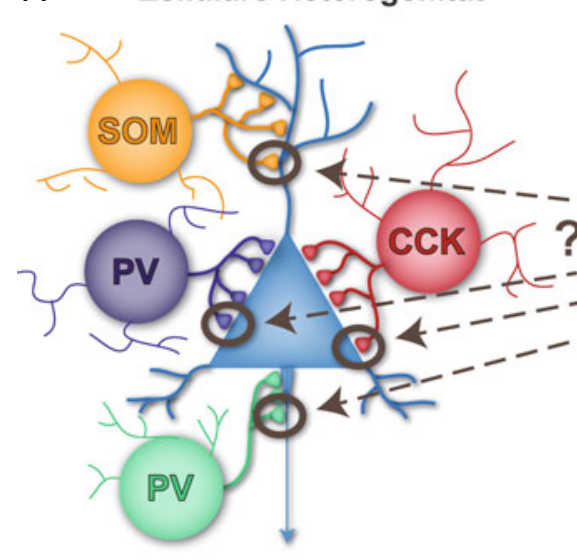

zen zu haben scheinen. So wurden z. B. Veränderungen in Gephyrin mit Schizophrenie und Autismus in Verbindung gebracht, Veränderungen in Collybistin dagegen mit Angststörungen und Lernbehinderungen [3]. Woher stammt diese Heterogenität? Die Ursache hierfür liegt vermutlich unter anderem in der außerordentlichen Komplexität des inhibitorischen Nervensystems. Es gibt Dutzende verschiedener Nervenzelltypen, die alle GABA freisetzen und hemmend wirken, sich aber grundlegend in ihren morphologischen, physiologischen und biochemischen Eigenschaften unterscheiden (Abb. 2A). Aufgrund dieser heterogenen Eigenschaften, die auch die synaptische Übertragung und ihre Konsequenzen beeinflussen, spielen sie sehr unterschiedliche Rollen in der Informationsverarbeitung im Gehirn [1, 2]. So kontaktieren beispielsweise Korbzellen, die durch die Expression des $\mathrm{Ca}^{2+}$-bindenden Proteins Parvalbumin (PV) identifiziert werden können, nur den perisomatischen Bereich am neuronalen Zellkörper, nicht aber die dendritischen Zellfortsätze, über die das Neuron exzitatorische Informationen von anderen Neuronen erhält. Dies führt zu einer stärkeren, aber weniger differenzierten Hemmung als die der Somatostatin(SOM)-exprimierenden GABAergen Neurone, welche die dendritischen Fortsätze kontaktieren und dort gezielt einzelne exzitatorische Inputs regulieren können. Während die zelluläre Heterogenität der GABAergen Neurone ausgiebig erforscht und gut verstanden ist, ist über eine mögliche molekulare Heterogenität der Proteinkomplexe an inhibitorischen Synapsen noch sehr wenig bekannt (Abb. 2A, B). Insbesondere stellt sich die Schlüsselfrage, ob alle neu iden-

B Molekulare Heterogenität?

$\Delta$ Abb. 2: Zelluläre und molekulare Heterogenität im inhibitorischen System. A, schematische Darstellung einiger Subtypen inhibitorischer Neurone, die unterschiedliche Bereiche des postsynaptischen Neurons kontaktieren und durch die Expression von Parvalbumin (PV), Somatostatin (SOM) oder Cholecystokinin (CCK) identifiziert werden können. B, Eine wichtige aktuelle Frage ist, ob die molekularen Komplexe an inhibitorischen Synapsen (im Detail in Abb. 1B beschrieben) ebenso heterogen sind wie die inhibitorischen Zelltypen.

tifizierten organisatorischen Proteine an allen inhibitorischen Synapsen agieren oder ob es hier eine molekulare Vielfalt gibt, die der zellulären Vielfalt des inhibitorischen Systems entspricht. Erste Hinweise deuten darauf hin, dass z. B. Nlgn2 nur perisomatische, nicht aber dendritische, Synapsen beeinflusst [4, 5], während Neuroligin-3 unterschiedliche Effekte an perisomatischen Synapsen von PV-positiven und Cholecystokinin(CCK)-positiven Neuronen hat [6]. Diese Heterogenität hat natürlich entscheidende Auswirkungen auf die Konsequenzen einzelner Mutationen für psychiatrisch relevante Verhalten. Für die Forschung stellt sie eine gewaltige Herausforderung dar, da jeder Synapsentyp einzeln in vivo in intakten neuronalen Schaltkreisen untersucht werden muss. Aus medizinischer Sicht ergibt sich daraus allerdings potenziell auch eine große Chance, da man die molekulare Vielfalt eventuell nutzen kann, um schaltkreisspezifische pharmakologische Ziele zu identifizieren, die therapeutische Eingriffe mit weniger unspezifischen und teilweise verheerenden Nebenwirkungen ermöglichen können.

\section{Inhibitorische Synapsen in Angstschaltkreisen}

Ein interessantes Beispiel für die Relevanz dieser Vielfalt zeigt sich in den neuronalen Schaltkreisen der Amygdala, die das Angstverhalten steuern. Die Amygdala ist eine mandelförmige Region im Temporallappen des Gehirns, die eine zentrale Rolle in der Verarbeitung von Emotionen spielt [2, 7]. Die Schaltkreise in der Amygdala umfassen ein komplexes Netzwerk verschiedener inhibitorischer Neurone, die sehr unterschiedliche 


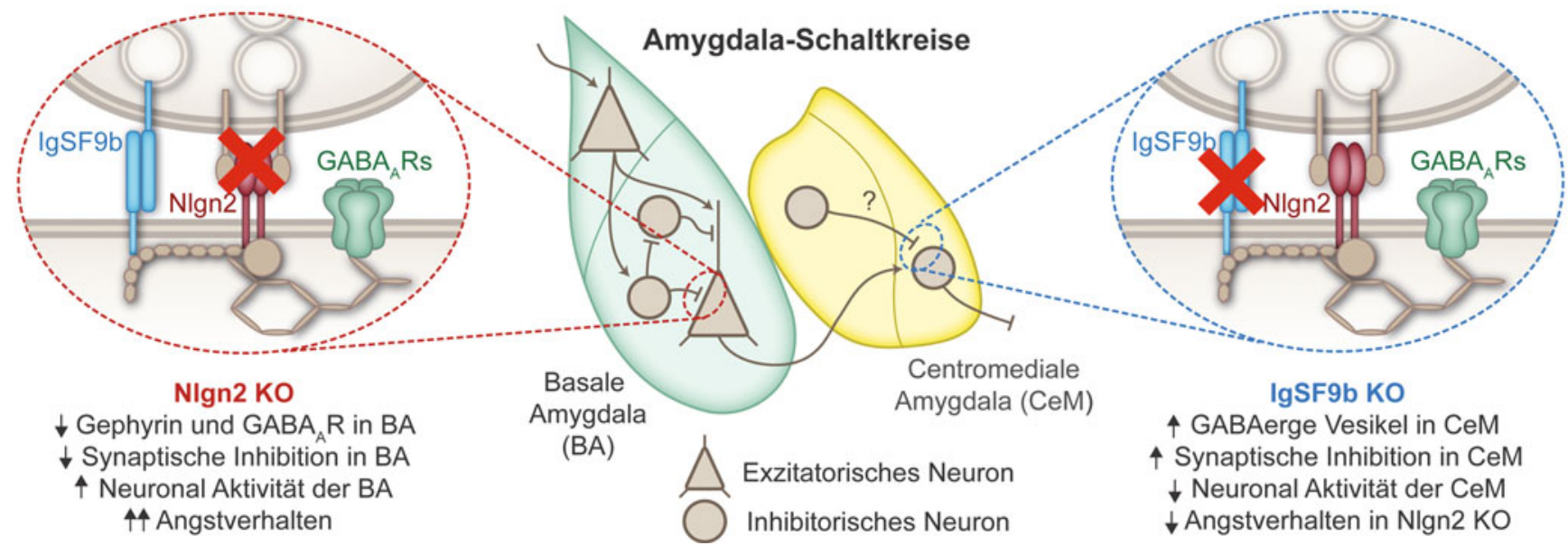

$\triangle$ Abb. 3: Heterogene Rollen von NIgn2 und IgSF9b in Angstschaltkreisen in der Amygdala [5, 9]. Mitte: vereinfachte Übersicht über Amygdala-Schaltkreise (aus [2]). Die basale Amygdala (BA) ist der Eingangskern, der Angst-relevante Informationen aus Cortex und Thalamus erhält. Die centromediale Amygdala (CeM) ist der Ausgangskern, der das resultierende Angstverhalten steuert. In beiden Kernen spielen inhibitorische Synapsen eine wichtige Rolle in der Informationsverarbeitung. Links: Der Verlust von Nlgn2 (Knock-out, KO) führt in der BA zu einer Reduktion in der Anzahl von Gephyrin- und

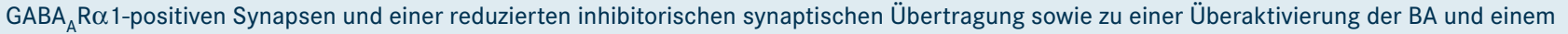
exzessiven Angstverhalten [5]. Rechts: Der Verlust von IgSF9b (KO) führt in der CeM zu einer Erhöhung der Anzahl oder Funktion präsynaptischer GABAerger Vesikel und somit der inhibitorischen synaptischen Übertragung sowie zu einer Normalisierung der CeM-Aktivierung und des Angstverhaltens in NIgn2-KO-Mäusen [9].

Funktionen in der Verarbeitung von Angstinformationen übernehmen (Abb. 3, Mitte). Dementsprechend können pharmakologische Wirkstoffe, die auf GABAerge Synapsen abzielen, je nach Synapsenspezifität sehr unterschiedliche Einflüsse auf das Angstverhalten haben. Dies ist besonders relevant für die Therapie von Angststörungen, die unter anderem mit positiven allosterischen Modulatoren der $\mathrm{GABA}_{\mathrm{A}} \mathrm{Rs}$, wie z. B. Benzodiazepinen, behandelt werden. Benzodiazepine sind effektive Anxiolytika, haben aber solch verheerende Nebenwirkungen außerhalb der Angstschaltkreise, dass sie im klinischen Alltag nur selten eingesetzt werden können [8]. Die Identifizierung pharmakologischer Ziele an spezifischen inhibitorischen Synapsen in den Schaltkreisen der Angst könnte dieses Problem lösen - mit dieser Fragestellung beschäftigt sich meine Arbeitsgruppe seit einigen Jahren [2, 3]. So konnten wir z. B. in Mausmodellen zeigen, dass der Verlust von Nlgn2 zu spezifischen Defiziten in der Signalübertragung an perisomatischen inhibitorischen Synapsen in der basalen, aber nicht zentralen Amygdala führt und dass dies mit einer Übererregung der Amygdala und einem exzessiven Angstverhalten einhergeht (Abb. 3, links, [5]). Dagegen führte der Verlust von IgSF9b, einem Adhäsionsprotein, das zuvor aus Zellkulturstudien als Interaktionspartner von Nlgn2 vermutet wurde, in Mäusen zu einem angstlösenden Effekt, der sich aus einer Verstärkung der inhibitorischen Signalübertragung an spezifischen Synapsen in der centromedialen Amygdala ergab (Abb. 3, rechts, [9]). Diese Ergebnisse deuten darauf hin, dass
IgSF9b-enthaltende Synapsen eventuell interessante Ziele für anxiolytische Therapien sind. Auch außerhalb der Amygdala spielen inhibitorische Synapsen eine wesentliche Rolle im Angstverhalten. So fanden wir heraus, dass der Verlust von Nlgn2 ebenfalls zu einer exzessiven Aktivierung des ventralen Hippocampus führt und dass vor allem der Transfer von Angstinformationen zwischen Gehirnregionen gestört war [10]. Unsere Ergebnisse, zusammen mit denen vieler anderer Gruppen, weisen darauf hin, dass ein besseres Verständnis der molekularen Heterogenität von organisatorischen Komplexen an inhibitorischen Synapsen einen entscheidenden Beitrag zur Entwicklung neuer therapeutischer Ansätze für Angststörungen und andere psychiatrische Erkrankungen liefern kann.

\section{Literatur}

[1] Marín O (2012) Interneuron dysfunction in psychiatric disorders. Nat Rev Neurosci 13:107-120

[2] Babaev O, Piletti Chatain C, Krueger-Burg D (2018) Inhibition in the amygdala anxiety circuitry. Exp Mol Med 50:18

[3] Krueger-Burg D, Papadopoulos T, Brose N (2017) Organizers of inhibitory synapses come of age. Curr Opin Neurobiol 45:66-77

[4] Poulopoulos A, Aramuni G, Meyer G et al. (2009) Neuroligin 2 drives postsynaptic assembly at perisomatic inhibitory synapses through gephyrin and collybistin. Neuron 63:628-642

[5] Babaev O, Botta P, Meyer E et al. (2016) Neuroligin 2 deletion alters inhibitory synapse function and anxiety-associated neuronal activation in the amygdala. Neuropharmacology 100:56-65

[6] Foldy C, Malenka RC, Sudhof TC (2013) Autism-associated neuroligin-3 mutations commonly disrupt tonic endocannabinoid signaling. Neuron 78:498-509

[7] Ehrlich I, Humeau Y, Grenier F et al. (2009) Amygdala inhibitory circuits and the control of fear memory. Neuron 62:757-771

[8] Bandelow B, Michaelis S, Wedekind D (2017) Treatment of anxiety disorders. Dialogues Clin Neurosci 19:93-107 [9] Babaev O, Cruces-Solis H, Piletti Chatain C et al. (2018) IgSF9b regulates anxiety behaviors through effects on centromedial amygdala inhibitory synapses. Nat Commun 9:5400 [10] Cruces-Solis H, Babaev O, Ali H et al. (2019) Altered theta/beta frequency synchrony links abnormal anxietyrelated behaviors to synaptic inhibition in Neuroligin-2 knockout mice. BioRxiv, doi: 10.1101/726190

Open Access:

This article is distributed under the terms of the Creative Commons Attribution 4.0 International License (http://creativecommons.org/licenses/by/4.0/), which permits use, duplication, adaption, distribution and reproduction in any medium or format, as long as you give appropriate credit to the original author(s) and the source, provide a link to the Creative Commons license, and

indicate if changes were made.
Open access funding provided by Max Planck Society.

Korrespondenzadresse:

PD Dr. Dilja Krueger-Burg

Abteilung Molekulare Neurobiologie

Max-Planck-Institut für Experimentelle Medizin

Hermann-Rein-Straße 3

D-37075 Göttingen

Tel.: 0551-3899-685

krueger@em.mpg.de

Dilja Krueger-Burg
1997-2001 Biochemiestudium an der Oxford University, UK. 2007 Promotion in
Neurowissenschaften an der Yale University, New Haven, USA. 2007-2010 Postdoc
am Massachusetts Institute of Technology, Cambridge, USA. 2010-2018 Postdoc und
anschließend Projektgruppenleiterin am Max-Planck-Institut für Experimentelle
Medizin in Göttingen. 2019 Habilitation in Biochemie, Universitätsmedizin Göttingen;
dort seit 2019 Gruppenleiterin an der Klinik für Psychiatrie.

